\title{
The Epidemiologists and the Epidemic Covid-19 in Cuba
}

\section{Silvia Martinez Calvo*}

Department of Hygiene and Epidemiology, National School of Public Health, Cuba

\begin{abstract}
*Corresponding author: Silvia Martinez Calvo, Consultant Professor, Department of Hygiene and Epidemiology, National School of Public Health, Calle 21 \# 3424 e/ 34 y 36, Municipio Playa. CP 11300, La Habana, Cuba, Email: calvo@infomed.sld.cu; elenuca17@gmail.com
\end{abstract}

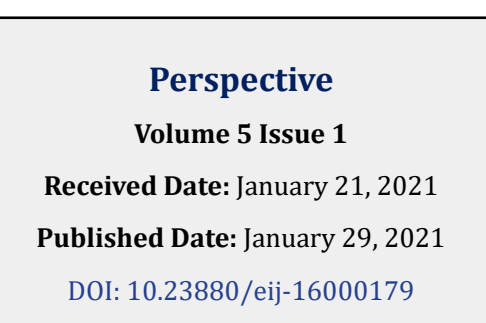

Keywords: Asymptomatic Person; Catastrophe; mobility; Burden of Viruses; Epidemic Evaluation.

\section{Introduction}

The mention of the term epidemic, both for the scientific community and for the population in general, provides an opportunity to recognize the role of the epidemiologist in any country, but after the critical situation ended, they return to the anonymous site of their work, at least in health services level. The COVID-19 epidemic has represented an opportunity to confirm or refuse this statement, although the functions and control activities carried out by epidemiologists for eliminate this epidemic have not always been widely recognized, because of the tendency to prioritize the medical care. The purpose of this brief comment is to describe some COVID-19 epidemic characteristics in Cuba, and the role of epidemiologists.

\section{The People and the SARS-CoV-2}

The epidemic process caused by an infectious agent implicates diverse individual responses and the SARS-CoV-2 is not exception, because this virus reproduces the known characteristics of a biological agent entirely, to produce the expected community response disease [1]. It includes asymptomatic carriers, nowadays defines like "asymptomatic person"; thus, it has privileged the control activities focus on COVID-19 symptoms, better than investigate the real possibility of transmission due to the "burden of viruses", that this persons spread silently, although it is suspected that "people are more contagious when they are more symptomatic (more sickest)" [2] and not as carriers asymptomatic.

A relevant issue related to the epidemiological concepts, is the use of the term "event" to replace "epidemic outbreak"; some Cuban researchers consider that this change minimize the possible "sensationalism" or impression of "catastrophe" produced by the word "epidemic outbreak" [3]. Whatever this argumentative reason, it would not be appropiate to modify or change basic categories of any science and it's better to explain their meaning to the people, crearly. Nevertheless, that internationally accepted definitions are needed for comparative purposes.

In the other hand, the importance given to the geographic space (georeferencing) for the control of the epidemic is another example to proof that it is well used for the exact location of cases in the respective population places (cities, towns, towns and villages), where the person acquired the disease (source of infection or contagious), and specify the route of their "mobility" (community spread) for better detection of contacts and suspects, without being restricted to the conventional political-administrative divisions of the territories [4].

\section{Epidemiologic Control of COVID-19 in Cuba}

The island of Cuba occupies a privileged geographical position between the North and South hemispheres in the Americas. The current population is 11 million and $20 \%$ belong to the age group over 60 years, an important vulnerable population group in relation to the epidemic. The health system has universal coverage, with more than 300,000 professionals, technicians and administrative personnel work [5]. A National Plan for the Control and Prevention of COVID-19 was established in January 2020; this plan included the Phases I-III that established the epidemic control, a strict epidemiological surveillance and healthcare services in hospitals and isolation centers for contacts and suspects persons [6]. The effectiveness of establishing isolation centers for contacts and suspects, which was discharged to hospitals for the sick, is undeniable.

The actions of the Cuban Government were undertaken from the beginning with an intersectorial approach and were carried out at the same time and in a uniform manner in all 


\section{Epidemiology International Journal}

the country's provinces, through local governments and state institutions [7]. This intersectorial work -another control measure- should not remain at the high or intermediate decision-makers, but rather it should be reproduced at the real local level, where its usefulness would be greater. Since epidemiological work has always required that essential interdisciplinary relations, groups of experts were integrated by epidemiologists, mathematical, geographers, microbiologists, psychologists and other specialists, without the predominance of some disciplines over others, but with an accurate selection for its leadership and an integral composition of the team, if the expected results were desired [8].

The introduction of mathematical models to predict the evolution of the epidemic, a necessary exercise, although not so novel for epidemiology, constitutes a good example of interdisciplinary work. Undoubtedly, this tools provided useful guides to implement the epidemiological control actions, but it should not be transformed into an oracle of permanent observation that relegate the field work in places where people live and work; ultimately, they are the same cases and suspects persons, who contribute to the incidence rates for these statistical predictions.

\section{Epidemic Evolution}

In Cuba, the SARS-Cov-2 epidemic has passed since its inception in March 2020, with epidemic outbreaks located in several territories, large areas of silence in the eastern part of the country and a large outbreak starting in October 2020, coinciding with the opening of airports. The spread of the virus has been confirmed in important investigations carried out during the development of the national epidemic, for example, to estimate the prevalence of infection by SARSCOV-2 based on the standard protocol suggested by the WHO for epidemiological studies of COVID-19 [9].

Until October 1, 2020, in Cuba 1 million 605 thousand 819 PCR tests for the diagnosis of SARS-CoV-2 have been carried out; 5670 cases of COVID-19 had been confirmed, a maximum rate of 7.43 per 100,000 inhabitants and 122 people had died (2.15), one of the lowest mortality rate in the Americas. In the same period, 625 cases of COVID-19 under the age of 18 had been detected in the country [7]. Although this number is greater than those reported internationally, in most of the children the disease progressed without complications or asymptomatic [10].

On the other hand, with regarding to sex, throughout the epidemic men have predominated with $63.9 \%$. With the new epidemic outbreak that started between OctoberNovember 2020, accumulated 14, 576 patients in the first weeks of January 2021, 1560 under 18 years, 151 patients died and 11,863 recovered patients accumulated (81.4\%) [11]. The information related to compulsory anti-epidemic activities has been plenty, and released on TV by the National Director of Epidemiology as the main spokesman, although some groups of population have inconsistent conduct to accomplish those activities.

One of the most positive experiences of the epidemic has been the multidisciplinary work promoted by epidemiologists, although they have not always been the main protagonists. Besides, the contribution of Cuban scientists for the treatment of the sick was important for reduce the mortality rate, but it's result must be evaluated rigoruosly, as well as the study of the clinical sequelae by COVID-19 in discharged patients.

\section{Conclusion}

The contributions of the field epidemiological research must also be recognized, because of it effective strategies to reduce the spread of the coronavirus and to prevent high level of morbidity and mortality of our population. The national epidemic derived from the global pandemic, should be considered as an opportunity to recognize first, the capacity of the cuban universal health system to serve the entire population; publicize the performance of epidemiologists at different levels of health services, further to announce the advances in the field of biomedicine which together with government strategies, should collaborated to reduce the consequences of COVID-19 in our population.

\section{References}

1. (2002) Module of Principles of Epidemiology for Disease Control Unit 2. 2nd (Edn.), Pan American Health Organization (PAHO/WHO), Washington DC, pp: 44.

2. (2020) The Coronavirus Prevention Handbook. 101 science based tips that could save your life. In: Zhou W, Hubei Science and Technology Press, Wuhan, China.

3. Peláez MP (2020) Outbreaks, epidemics, events and other epidemiological terms of daily use. Cuban Journal of Public Health 46(2): 2358.

4. Iñiguez L (2020) Space and territory in the analysis of the health situation. In: Martínez S (Editor.), Health situation analysis. A new look. Chapter 4. Havana, Medical Sciences.

5. (2019) Republic of Cuba. Ministry of Public Health. Statistical Yearbook.

6. (2020) Republic of Cuba, Ministry of Public Health. COVID-19 prevention and control plan, strategy to be 


\section{Epidemiology International Journal}

properly and timely prepared. Havana: MINSAP.

7. Bermejo PM, Valdés LS, López LS, Valdivia Onega NC, Vidal Ledo MJ, et al. (2020) Equity and response of the Cuban National Health System to COVID-19. Pan American Journal of Public Health 44: 138.

8. Más P, Vidal M, Baldoquin W, Seuc AH, Guinovart R, et al. (2020) Organization of epidemiological research for the epidemic fight against COVID-19 in Cuba. Infodir 32: 831.
9. Juanes WS (2020) Cuba, the first country in the Americas to conduct a COVID-19 incidence study. Granma.

10. López L, Noda A, Castro B, Cruz SM, Causa N, et al. (2020) Clinical epidemiological characterization of 183 Cuban children with SARs-COV2 infection. Cuban Journal of Pediatrics 92: 1256.

11. (2020) Republic of Cuba. Ministry of Public Health. Coronavirus in Cuba. MINSAP. 